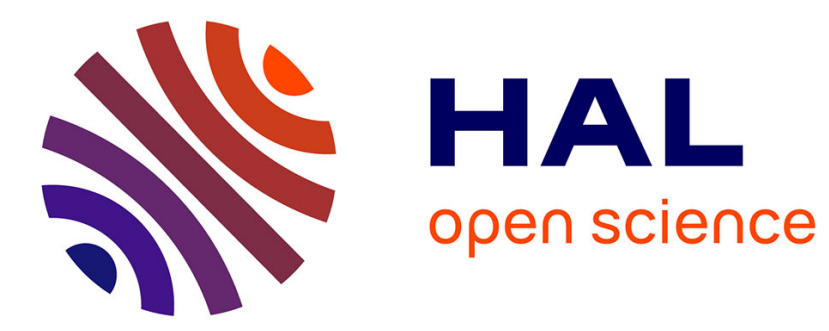

\title{
A Radon-Nikodym approach to measure information
} Yann Rébillé

\section{To cite this version:}

Yann Rébillé. A Radon-Nikodym approach to measure information. 2010. hal-00526251

\section{HAL Id: hal-00526251 \\ https://hal.science/hal-00526251}

Preprint submitted on 15 Oct 2010

HAL is a multi-disciplinary open access archive for the deposit and dissemination of scientific research documents, whether they are published or not. The documents may come from teaching and research institutions in France or abroad, or from public or private research centers.
L'archive ouverte pluridisciplinaire HAL, est destinée au dépôt et à la diffusion de documents scientifiques de niveau recherche, publiés ou non, émanant des établissements d'enseignement et de recherche français ou étrangers, des laboratoires publics ou privés. 

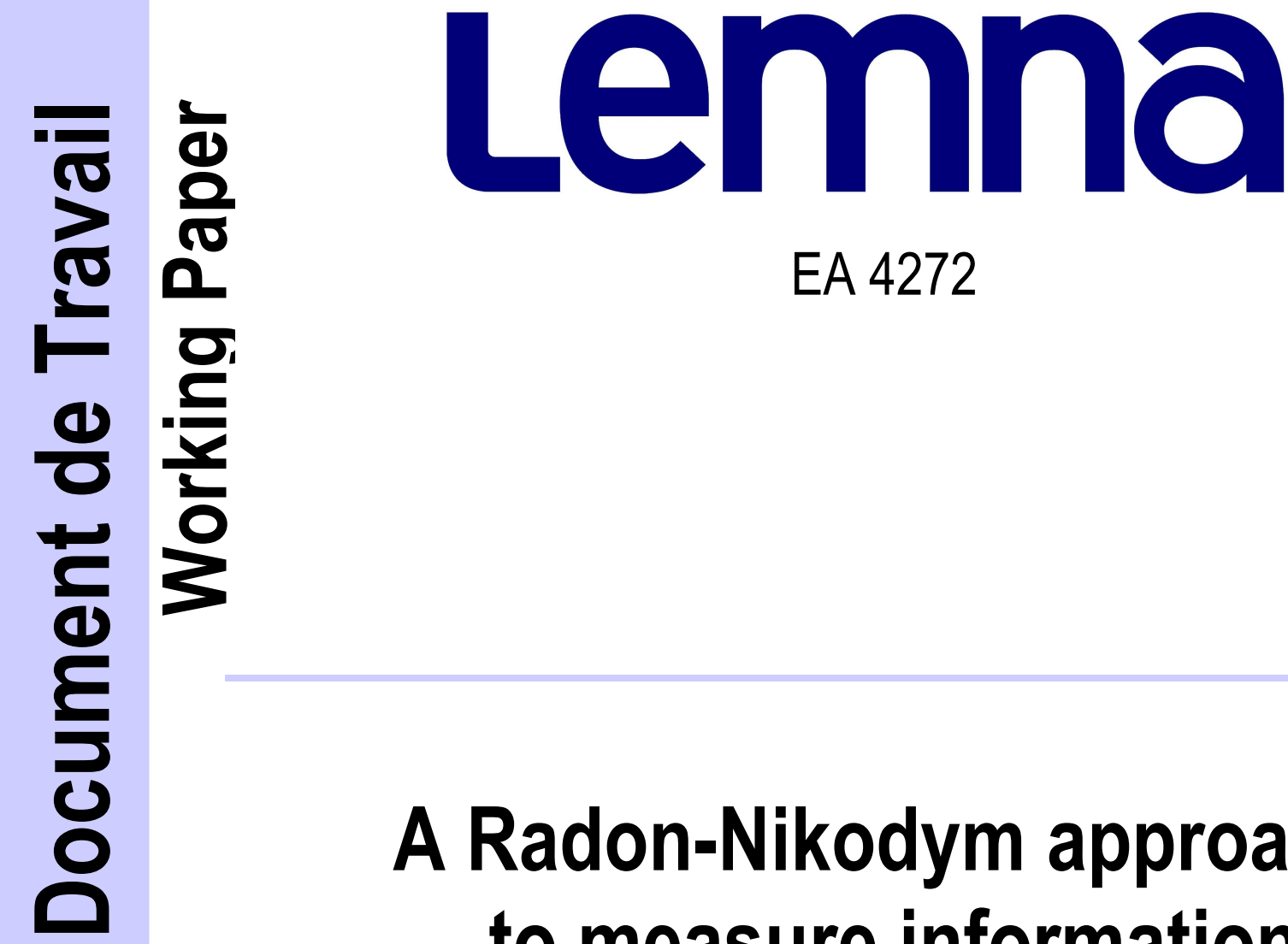

EA 4272

\title{
A Radon-Nikodym approach to measure information
}

\author{
Yann Rébillé ( ${ }^{*}$ )
}

\author{
$2010 / 21$
}

$\left.{ }^{*}\right)$ LEMNA - Université de Nantes

Laboratoire d'Economie et de Management Nantes-Atlantique

Université de Nantes

Chemin de la Censive du Tertre - BP 52231

44322 Nantes cedex 3 - France

www.univ-nantes.frliemn-iae/recherche

Tél. +33 (0)2 40141717 - Fax +33 (0)2 40141749 


\title{
A Radon-Nikodym approach to measure information
}

\author{
Yann Rébillé * \\ LEMnA, Institute of Economics and Management of Nantes-IAE, \\ chemin la Censive du Tertre, BP 52231, 44322 Nantes Cedex 3, France.
}

\begin{abstract}
We consider a decision maker facing uncertainty which behaves as a subjective expected utility maximizer. The value of information is traditionnaly captured as a greater expected utility the decision maker can achieve by selecting a best strategy as information arrives. We deal with the limit process of being better informed and introduce an information density function depending soley on the states that gives an exact least upper bound to being more informed. This information density function is given by a Radon-Nikodym's type theorem for set functions and is explicitely computed for the countable case.
\end{abstract}

Keywords: decision making under uncertainty, value of information, expected utility, capacity, Radon-Nikodym derivative.

JEL Classification: D 80, D 82, C 71.

\section{Introduction}

Traditionnaly more information is valuable for the decision maker (DM). As information becomes available the decision making process can be improved. Using Savage's words (see 1.14 p.107 in [10]) "knowledge is not disavantageous". Our main interest is to consider the limit process of being better informed and how to measure the overall information the DM could benefit from.

We consider a decision maker facing uncertainty which behaves as a subjective expected utility maximizer ([10]). Typically, whenever information is obtained

*Yann.Rebille@univ-nantes.fr 
through a simple message, i.e. a partition ([1]), then the DM can choose a best strategy on each atom and therefore raises its overall ex-ante expected utility ([4]). Since information is a desirable good, a DM can be willing to undergo some cost in order to gather more information (see [6]). It seems therefore natural to raise the following question. How much utility can the DM expect as he gathers more and more information? For this matter we will produce an information density function depending soley on the states, that can be interpreted as an indirect utility for states. This information density function gives an exact upper bound to being fully informed. It is obtained by means of a general RadonNikodym's type theorem for monotone subadditive set functions of bounded sum with respect to measures as treated in an earlier work $([9])$.

\section{$1.1 \quad$ Framework}

Let $\Omega$ denote the set of states. A DM is uncertain about the true state that will prevail. Let $S$ be a non-empty set of strategies available to the DM. We shall assume that there exists a bounded utility function (see Theorem 14.5 p.206 in [3] ${ }^{1}$

$$
u: S \times \Omega \longrightarrow[0, M]
$$

and that each strategy is evaluated in state $\omega$ through $u(s, \omega)$. Hence each strategy $s$ is evaluated through its expected utility ${ }^{2}: \forall s, s^{\prime} \in S$

$$
s \succeq s^{\prime} \Longleftrightarrow \int_{\Omega} u(s, \omega) d \mu(\omega) \geq \int_{\Omega} u\left(s^{\prime}, \omega\right) d \mu(\omega)
$$

We assume without loss of generality that there exists a null-strategy $s_{0} \in S$ such that

$$
u\left(s_{0}, \omega\right)=0, \mu-\text { a.e. }
$$

The null-strategy is the unique strategy available to the DM when no choice is made possible.

We consider now a simple situation where the set of strategy $S$ is not always available, and depends on the realization of some event $A \in \mathcal{A}$. The DM is ask to choose some strategy $s$ if $A$ occurs, that is to say if $A$ includes the true state. Otherwise if $A^{c}$ occurs the DM has no other choice than $s_{0}$. In this case, the expected utility of strategy $s$ conditionally on $A$ is given by

$$
\int_{A} u(s, \omega) d \mu(\omega)+\int_{A^{c}} u\left(s_{0}, \omega\right) d \mu(\omega)=\int_{A} u(s, \omega) d \mu(\omega)
$$

The capacity of $A$ is given by

$$
\operatorname{Cap}(A)=\sup _{S} \int_{A} u(s, \omega) d \mu(\omega)
$$

\footnotetext{
${ }^{1}$ In Savage's terminology, each couple $(s, \omega)$ can be interpreted as a consequence that is measured by a utility function and each strategy $s$ can be identified with a decision i.e., a mapping $s: \omega \mapsto(s, \omega)$.

${ }^{2}$ For any $s, u(s,$.$) is assumed to be measurable.$
} 
hence $\operatorname{Cap}(A)$ is the highest utility the DM can expect if he is capable to choose a strategy conditionally on $A$. Cap is a subadditive monotone set function (see Appendix B.1). This supremum condition becomes a maximum under suitable topological conditions ([5] and Appendix A.1).

By construction, the decision maker is always better off when he has the possibility to choose his strategy conditionaly on $A$ since he can always neglect his available choices by choosing $s_{0}$, i.e. $\operatorname{Cap}(A) \geq \int_{A} u\left(s_{0}, \omega\right) d \mu(\omega)=0$. Using Savage's words (see 1.12 p.107 in [10]) "the person is free to ignore the observation".

\subsection{Information}

Assume that the DM can be informed before the realization of uncertainty where the true state will lie in. For instance he can perform some experiment before the true state is revealed, and according to the message he receives he can therefore localize the true state and will choose a best strategy accordingly.

Formally speaking the DM faces a partition of $A$ and knows which atom of the partition will contain the true state and on each atom he chooses a strategy in order to maximize his expected utility, otherwise if $A^{c}$ occurs the DM has no other choice than $s_{0}$.

For a partition $\Pi_{A}=\left\{A_{k}\right\}_{k=1}^{n}$ of $A$, the highest expected utility that can be attained by the DM is given by

$$
\operatorname{Cap}\left(\Pi_{A}\right)=\sup _{\left(s_{1}, \ldots, s_{n}\right) \in S^{n}} \sum_{k=1}^{n} \int_{A_{k}} u\left(s_{k}, \omega\right) d \mu(\omega)
$$

or equivalently by separability of the expected utility functional,

$$
\operatorname{Cap}\left(\Pi_{A}\right)=\operatorname{Cap}\left(A_{1}\right)+\ldots+\operatorname{Cap}\left(A_{n}\right) .
$$

This specific property of additive separability of the information function ( $\Pi \mapsto$ $\operatorname{Cap}(\Pi))$ is axiomatically characterized in $[4,7]$.

Now if this process is carried on at the limit, the maximal utility he can extract from the information given by $A$ is ${ }^{3}$,

$$
I(A)=\sup _{\Pi_{A}} \operatorname{Cap}\left(\Pi_{A}\right) .
$$

The information gap at $A$ can be evaluated through the quantity,

$$
I G(A)=I(A)-\operatorname{Cap}(A), I G(A) \geq 0 .
$$

By construction,

$$
I(A) \leq M \mu(A)=\int_{A} M d \mu, \forall A \in \mathcal{A} .
$$

\footnotetext{
${ }^{3}$ The limit is taken with respect to the relation "finer than", i.e. $\Pi \geq \mathrm{R}$ if $\forall A \in \Pi, \exists B \in \mathrm{R}$ $/ A \subset B$.
} 
Our aim is to know if whether or whether not there is an upper bound to being better informed, besides the trivial constant function $M^{4}$. More precisely we wish to know if there exists an information density function $U: \Omega \longrightarrow \mathbb{R}^{+}$(i.e. an integrable function) such that for all $A \in \mathcal{A}$,

$$
I(A) \leq \int_{A} U(\omega) d \mu(\omega) .
$$

Moreover, the information density function $U$ should be minimal, that is, for any integrable measurable $V: \Omega \longrightarrow \mathbb{R}^{+}$,

$$
\forall A \in \mathcal{A}, \operatorname{Cap}(A) \leq \int_{A} V(\omega) d \mu(\omega) \Rightarrow U \leq V, \mu \text { a.s. }
$$

From the standpoint of measure theory, the decision making problem can be described through a probability measure $\mu$, a constant $M \geq 0$ and a set function $w$ satisfying,

$$
w \leq M \mu
$$

Indeed consider $\mathcal{M}_{S}=\left\{m_{s}: s \in S\right\}$, where

$$
m_{s}: \mathcal{A} \longrightarrow[0, M]: A \mapsto \int_{A} u(s, \omega) d \mu(\omega)
$$

and

$$
w:=\sup _{m \in \mathcal{M}_{S}} m=\sup _{S} m_{s}=\text { Cap }
$$

The existence of a minimal integrable function is made possible by a RadonNikodym theorem for the set function $w$ w.r.t. the measure $\mu$, which is precisely its Radon-Nikodym superior derivative (see [9]).

This allows us to give a positive answer to the existence of an information density and a way to compute it (see Appendix B.1).

Positive result: The information density function is given by the superior Radon-Nikodym derivative of Cap w.r.t. $\mu$ and is precisely the supremum of the Radon-Nikodym derivatives of the $\left\{m_{s}\right\}_{s}$, i.e., $u(s,$.

$$
U=\frac{d \mathrm{Cap}^{s}}{d \mu}=\bigvee_{s \in S} \frac{d m_{s}}{d \mu}=\bigvee_{s \in S} u(s, .)
$$

Morerover, the information set function can be factorized in a minimal way through $U$

$$
\forall A \in \mathcal{A}, I(A)=\int_{A} U(\omega) d \mu(\omega)
$$

whence $U=\frac{d I}{d \mu}$, the classical Radon-Nikodym derivative of $I$ with respect to $\mu$ and $\forall A \in \mathcal{A}$,

$$
\operatorname{Cap}(A)>0 \Longleftrightarrow I(A)>0 \Longleftrightarrow \mu(A \cap\{U>0\})>0 .
$$

\footnotetext{
${ }^{4}$ As for the converse situation, any bounded measurable function $U: \Omega \longrightarrow \mathbb{R}^{+}$can be trivially interpreted as the information density function derived from the degenerate decision problem where $S=\{1\}$ and $u(1, \omega)=U(\omega)$.
} 
As it turns out, information $I$ is in fact a measure which is dominated by $\mu$. Thus, the information gap, i.e. $I G=I$ - Cap, is a superadditive set function since Cap is subadditive and $I$ is additive.

The first equivalence is natural and states that information has a positive measure if and only if it is valuable to the DM. The second equivalence reduces information to its information density function. Hence, an atom is valueless as soon as is does not meet the support of the information function, and a state of nature $\omega$ that is relevant for the DM is informative whenever $U(\omega)>0$.

In the following section we elicitate the information density function for the countable case. Then, we give some examples that show various aspects of the information density function and make a comment on the entropy. Finally, we study a two-period model where information can be traded. We provide subjective upper/lower bounds for a DM's willingness to pay/accept additional information. An Appendix gathers all the proofs and the technical material related to superior Radon-Nikodym derivatives.

\section{Information density functions}

Besides the trivial constant function $M$ as an upper bound, one can also consider the utility of perfect information using the supremum function

$$
V(\omega)=\sup _{s \in S} u(s, \omega)
$$

that suggests to choose the best strategy accordingly with each state (see [5]). When $\Omega$ is countable, $V$ is an appropriate choice. However, when $\Omega$ is uncountable, utility of perfect information is not an appropriate choice and this motivates the use of an information density function instead. Both coincide when $\Omega$ is countable.

\subsection{The countable case}

The countable case: Let $\Omega$ be countable. We may assume w.l.o.g. that $\Omega=$ $\mathbb{N}, \mathcal{A}=2^{\mathrm{N}}$ and $\mu(\{\omega\})>0$. In this case the information density function is precisely

$$
U(\omega)=\sup _{S} u(s, \omega)
$$

and

$$
\begin{gathered}
\operatorname{Cap}(\{\omega\})=\mu(\{\omega\}) \times \sup _{S} u(s, \omega), \\
I(A)=\sum_{\omega \in A} \operatorname{Cap}(\{\omega\})=\sum_{\omega \in A} \mu(\{\omega\}) \times \sup _{S} u(s, \omega)=\int_{A} U d \mu
\end{gathered}
$$


So whenever $\Omega$ is countable the choice of a best strategy can be performed statewise (see Appendix B.2).

\subsection{The uncountable case: examples}

But when $\Omega$ is uncountable $V$ might not be minimal nor measurable and a fortiori not available to the DM. Let us now consider the case when $\Omega$ is uncountable. Take $\Omega=S=[0, \infty)$. Define $u$ by,

$$
\begin{aligned}
u:[0, \infty) \times[0, \infty) & \longrightarrow[0,1] \\
(s, \omega) & \mapsto \begin{cases}\frac{1}{1+s}, & \text { if } s=\omega \\
0, & \text { otherwise } .\end{cases}
\end{aligned}
$$

Define,

$$
\forall \omega \in \Omega, V(\omega)=\sup _{s \in S} u(s, \omega)=\frac{1}{1+\omega}
$$

and

$$
\forall \omega \in \Omega, U(\omega)=\sum_{n} \frac{1}{1+n} 1_{\{n\}}(\omega),
$$

where $1_{\{n\}}$ denotes the indicator function at $n$. We will consider the discrete probability measure $\mu$, defined by

$$
\forall n \in \mathbb{N}, \mu(\{n\})=(1-\delta) \delta^{n}
$$

with $\delta \in(0,1)$.

Example 1: We endow $\Omega$ with its Borel $\sigma$-algebra, $\mathcal{B}$.

$V$ is $\mathcal{B}$-measurable, since $\{V \geq t\}=\left[0, \frac{1-t}{t}\right]$ for $t \in(0,1]$.

Then,

$$
\operatorname{Cap}(A)=\sup _{S} \int_{A} u(s, \omega) d \mu(\omega)=\max _{n \in A} \frac{1}{1+n}(1-\delta) \delta^{n}=\frac{1}{1+n_{A}}(1-\delta) \delta^{n_{A}}
$$

where $n_{A}=\min \{n: n \in A\} . \operatorname{And}^{5}$,

$$
I(A)=\sum_{n \in A} \frac{1}{1+n}(1-\delta) \delta^{n}
$$

Since $\mu$ is concentrated on $\mathbb{N}$ we can pick as an information density function

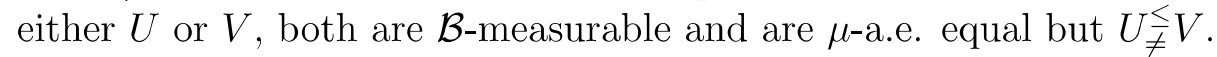

Let us introduce a particular $\sigma$-algebras to show that $V$ is not necessarily measurable.

Example 2: We endow $\Omega$ with the $\sigma$-algebra of countable-cocountable sets $\mathcal{A}_{\text {coc }}$.

$$
{ }^{5} I(\Omega)=\sum_{n} \frac{1}{1+n}(1-\delta) \delta^{n}=\frac{1-\delta}{\delta} \ln \left(\frac{1}{1-\delta}\right) \rightarrow 0(1) \text { when } \delta \rightarrow 1(0) .
$$


$A \in \mathcal{A}_{\mathrm{COc}}$ if $A$ is countable or if its complement $A^{c}$ is countable. For all $\omega$, $\{\omega\} \in \mathcal{A}_{\mathrm{coc}}$.

Now, $V$ is not $\mathcal{A}_{\text {coc-measurable, since }}\{V \geq t\}=\left[0, \frac{1-t}{t}\right]$ for $t \in(0,1]$ which is neither countable nor cocountable.

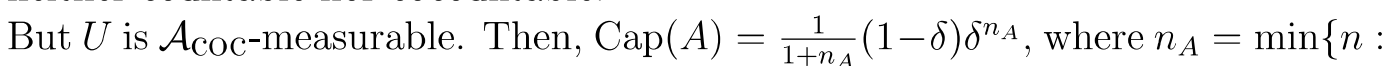
$n \in A\}$. And, $I(A)=\sum_{n \in A} \frac{1}{1+n}(1-\delta) \delta^{n}$. Hence the information density function is given by the classical Radon-Nikodym derivative of $I$ with respect to $\mu$ :

$$
U=\sum_{n} \frac{1}{1+n} 1_{\{n\}}=\frac{d I}{d \mu}
$$

Let us introduce another probability measure to show that $V$ is not necessarily minimal despite being measurable.

Example 3: We endow $\Omega$ with its Borel $\sigma$-algebra, $\mathcal{B}$.

We will consider the diffuse probability measure $\mu$, defined by

$$
\forall A \in \mathcal{B}, \mu(A)=\int_{A} \theta e^{-\theta x} d \lambda(x)
$$

with $\theta>0$ and $\lambda$ the Lebesgue measure on $\mathbb{R}^{+}$.

Since $u(s, \omega)=0, \lambda$-a.e for all $s$ and $\mu$ is diffuse then for all $A \in \mathcal{B}, \int_{A} u(s,). d \mu=$ 0 . Hence $U=0$, Cap $=0, I=0$ but $\mu(\{V>U\})=1$. So clearly $V$ is not minimal despite being measurable.

\subsection{Entropy: a comment}

Information that can be extracted from a simple random experiment such as a partition is widely associated to the notion of entropy.

The partition-entropy of $\Pi=\left\{A_{i}\right\}_{i=1}^{n}$ is defined by,

$$
h(\Pi)=-\sum_{i=1}^{n} \mu\left(A_{i}\right) \ln \left(\mu\left(A_{i}\right)\right)
$$

By extension one could define the maximal-entropy by,

$$
H=\sup _{\Pi} h(\Pi)
$$

A naive question is the following,

"Is there a decision making under uncertainty problem such that partition-entropy could be interpreted as the capacity of a partition and maximal-entropy as information?"

The answer is twice no. The first objection is quite obvious and can be overcomed. The second is more definitive. Let $\mu$ be a non-atomic probability measure on $\mathcal{A}$ and

$$
f:[0,1] \longrightarrow \mathbb{R}^{+}: p \mapsto-p \ln (p)
$$


with $f(0)=0$. Put $w=f \circ \mu$. Hence,

$$
h(\Pi)=-\sum_{i=1}^{n} \mu\left(A_{i}\right) \ln \left(\mu\left(A_{i}\right)\right)=\sum_{i=1}^{n} w\left(A_{i}\right)
$$

however, $w$ is not a monotone set function, since $w(\emptyset)=w(\Omega)=0$, so $w$ can not play the rôle of a capacity ${ }^{6}$.

The second objection is without recourse. Since $\mu$ is non-atomic for all $n$ there is a partition $\Pi_{n}=\left\{A_{i}\right\}_{i=1}^{n}$ such that $\mu\left(A_{i}\right)=\frac{1}{n}$ and $\sum_{i=1}^{n} A_{i}=\Omega$. Hence,

$$
h\left(\Pi_{n}\right)=\sum_{i=1}^{n} f\left(\mu\left(A_{i}\right)\right)=n f\left(\frac{1}{n}\right)=-\ln \left(\frac{1}{n}\right)=\ln (n) \uparrow \infty .
$$

Thus, $H={ }_{\text {def }} \sup _{\Pi} h(\Pi) \geq \sup _{n} h\left(\Pi_{n}\right)=+\infty$. So $w$ can not be majorized by an information density function and a fortiori by any constant $M$, i.e., $\forall A \in$ $\mathcal{A}, w(A) \leq M \mu(A)$.

\section{WTP/WTA for information}

We consider a two-period ${ }^{7}$ model with date $t=1,2$. The decision maker possesses a monetary amount $m>0$ at date $t=1$ and an information partition $\Pi$ which enables him to choose a best strategy at $t=2$. We assume that the DM has an additive time-separable utility function, i.e.

$$
u(m,(s, \omega))=u_{1}(m)+u_{2}(s, \omega)
$$

with $u_{1}$ differentiable, increasing and that he exhibits a decreasing marginal utility for wealth, i.e., $u_{1}$ is concave. So its expected utility given $(m, \Pi)$ is,

$$
u_{1}(m)+\operatorname{Cap}_{u_{2}}(\Pi)
$$

At $t=1$, the DM is offered the possibility to purchase some additional information $\Pi^{\prime}$ at cost $p$ that allows him to modify its earlier strategy. Hence its expected utility given $\left(m-p, \Pi \vee \Pi^{\prime}\right)$ is ${ }^{8}$,

$$
u_{1}(m-p)+\operatorname{Cap}_{u_{2}}\left(\Pi \vee \Pi^{\prime}\right)
$$

So an additional information is worthwhile whenever

$$
u_{1}(m-p)+\operatorname{Cap}_{u_{2}}\left(\Pi \vee \Pi^{\prime}\right) \geq u_{1}(m)+\operatorname{Cap}_{u_{2}}(\Pi)
$$

\footnotetext{
${ }^{6}$ We could have consider the monotone extension of $f$ but a fortiori the maximal-entropy would be infinite.

${ }^{7}$ Our model is different from La Valle's [6] where a one-period model is introduced dealing necessarily with monetary outcomes.

${ }^{8} \Pi \vee \Pi^{\prime}$ is the coarsest partition which is finer than $\Pi$ and $\Pi^{\prime}$.
} 
Symetrically, a DM, with initial wealth and information $\left(m, \Pi \vee \Pi^{\prime}\right)$, is willing to abandon some information $\Pi^{\prime}$ at some price $p$ if,

$$
u_{1}(m+p)+\operatorname{Cap}_{u_{2}}(\Pi) \geq u_{1}(m)+\operatorname{Cap}_{u_{2}}\left(\Pi \vee \Pi^{\prime}\right)
$$

The information set function provides a way to obtain an upper bound for the DM's willingness to pay given $(m, \Pi)$ for any additional information $\Pi^{\prime}$. Let us call it the absolute willingess to pay (AWTP), defined by,

$$
\Phi_{u}(m, \Pi)=\frac{1}{u_{1}^{\prime}(m)}\left[I_{u_{2}}(\Omega)-\operatorname{Cap}_{u_{2}}(\Pi)\right]
$$

where $I_{u_{2}}$ is the information set function derived from the utility at date $t=2$. The AWTP is an upper bound for the WTP to be fully informed. This quantity can be loosely interpreted as a maximal marginal rate of substitution: where the DM abandon one unit of util at date 1 at a "price" of $u_{1}^{\prime}(m)$ for a maximal gain in utility at date $t=2: I_{u_{2}}(\Omega)-\operatorname{Cap}_{u_{2}}(\Pi)$, the information gap ${ }^{9}$.

$\Phi_{u}$ is cardinal, non-increasing with respect to information by construction and non-decreasing with respect to wealth (see Appendix B.3).

In term of comparative statics, one can deduce that preference for the present decreases the AWTP. Consider the pairs $\left(u_{1}, u_{2}\right)$ and $\left(v_{1}, u_{2}\right)$. Then ${ }^{10}$,

$$
\Phi_{\left(u_{1}, u_{2}\right)}(m, \Pi) \geq \Phi_{\left(v_{1}, u_{2}\right)}(m, \Pi) \Longleftrightarrow u_{1}^{\prime}(m) \leq v_{1}^{\prime}(m)
$$

In particular, if $v_{1}=(1+\delta) u_{1}$ with $\delta>0$, then the AWTP decreases with $\delta$.

More generally, the AWTP can be decomposed setwise with respect to $\Pi$ (see Appendix B.3). Let $\Pi=\left\{A_{1}, \ldots, A_{K}\right\}$. It holds,

$$
\Phi_{u}(m, \Pi)=\sum_{k=1}^{K} \Phi_{u}\left(m, A_{k}\right)
$$

where

$$
\Phi_{u}\left(m, A_{k}\right)=\frac{I_{u_{2}}\left(A_{k}\right)-\operatorname{Cap}_{u_{2}}\left(A_{k}\right)}{u_{1}^{\prime}(m)} .
$$

$\Phi_{u}\left(m, A_{k}\right)$ is the DM's AWTP to pay for being more informed whenever $A_{k}$ occurs.

Finally, the function $\Phi$ allows us to give some bounds for the willingness to pay or to accept some additional information (see Appendix B.3). For all $\left(m, \Pi, \Pi^{\prime}\right)$,

$$
W T P \leq \Phi_{u}(m, \Pi)-\Phi_{u}\left(m, \Pi \vee \Pi^{\prime}\right) \leq W T A .
$$

The first inequality says that in order to acquire full information one should proceed step by step: $W T P+\Phi_{u}\left(m, \Pi \vee \Pi^{\prime}\right) \leq \Phi_{u}(m, \Pi)$.

The second says that the DM is always willing to be better informed, since he is still willing to pay more when he possesses more information than he could with less and having sold a piece of information: $\Phi_{u}(m, \Pi)-W T A \leq \Phi_{u}\left(m, \Pi \vee \Pi^{\prime}\right)$.

\footnotetext{
${ }^{9}$ When the marginal utility at date 1 is constant and equals to $1, u_{1}^{\prime}=1$, i.e. $u_{1}+u_{2}$ is quasi-linear, then the AWTP for more information is precisely the information gap.

${ }^{10}$ This condition on marginal utilities is independent of being "more/less risk averse than".
} 


\section{Conclusion}

In this article we adressed the question of how a subjective expected utility maximizer would evaluate full information. We provide a way to compute information with an information density function that can be built starting with the utility/strategy set of the decision maker. Information density function departures from the "first guess" utility of perfect information which seems a natural pick but turns out to be inappropriate in general situation. This new approach uses intensively non-additive measures and a Radon-Nikodym theorem for subadditive set functions (TU-games). Information is thus modelized as a measure and allows us to introduce some tractable bounds for evaluating willingness to pay/accept for being better informed.

\section{A Technical material}

\section{A.1 Topological conditions}

The condition of maximality can be achieved under suitable topological conditions (see [5] for stronger conditions).

Proposition A.1 Let $S$ be a compact topological space that satisfies the first axiom of countability (e.g. $S$ is a metric compact space) and $u \geq 0$.

Assume that $\mu$-a.e., $u(., \omega)$ is upper semi-continuous (usc) and that $u(s,$.$) is$ integrable (for instance if $u \leq M$ ) then the maximum is attained.

\section{Proof.}

Let us show that

$$
F_{A}: S \longrightarrow \mathbb{R}: s \mapsto \int_{A} u(s, \omega) d \mu(\omega)
$$

is usc for all $A \in \mathcal{A}$. Hence by Weierstrass theorem the maximum will be attained on $S$ compact.

Now since $S$ is satisfies the first axiom of countability, we can prove that $F_{A}$ is usc by mean of sequences.

By hypothesis, there is a set $N \in \mathcal{A}$ with $\mu(N)=0$ such that $\forall \omega \notin N, u(., \omega)$ is usc.

Let $\left\{s_{n}\right\}_{n} \subset A$ be a sequence converging to $s \in S$. Put $f_{n}(\omega)=u\left(s_{n}, \omega\right), f(\omega)=$ $u(s, \omega)$, by hypothesis $0 \leq f_{n}(\omega), f(\omega) \leq M$ and $f_{n}, f$ are mesurable.

Since $u(., \omega)$ is usc for $\omega \notin N$, we have $\overline{\lim }_{n} f_{n}(\omega) \leq f(\omega)$. Thus,

$$
\begin{gathered}
\overline{\lim }_{n} F_{A}\left(s_{n}\right)=\overline{\lim }_{n} \int_{A} u\left(s_{n}, \omega\right) d \mu(\omega)=\overline{\lim }_{n} \int_{A} f_{n}(\omega) d \mu(\omega) \\
=\overline{\lim }_{n} \int_{A \backslash N} f_{n}(\omega) d \mu(\omega) \leq \int_{A \backslash N} \overline{\lim }_{n} f_{n}(\omega) d \mu(\omega) \leq \int_{A \backslash N} f(\omega) d \mu(\omega) \\
=\int_{A} f(\omega) d \mu(\omega)=\int_{A} u(s, \omega) d \mu(\omega)=F_{A}(s)
\end{gathered}
$$

The first inequality holds by Fatou's Lemma, the second by monotonicity. 


\section{A.2 Superior Radon-Nikodym derivatives}

From now on $(\Omega, \mathcal{A})$ denotes a measurable space.

$w: \mathcal{A} \longrightarrow \mathbb{R}^{+}$with $w(\emptyset)=0$ is a set function.

$w$ is monotone if $\forall A, B \in \mathcal{A}, w(A) \leq w(B)$ whenever $A \subset B$.

$w$ is null hereditary $(0-\mathrm{H})$ if $\forall A, B \in \mathcal{A}, w(A)=0 \Rightarrow w(B)=0$ whenever $B \subset A$. $w$ is subadditive if $\forall A, B \in \mathcal{A}, w(A \cup B) \leq w(A)+w(B)$ whenever $A \cap B=\emptyset$. $w$ is superadditive whenever the opposite inequality always holds.

$w$ is of bounded sum i.e., $w \in B S$ (see [8]) if

$$
\sup \left\{\sum_{i} w\left(P_{i}\right):\left\{P_{i}\right\}_{i} \in \mathcal{P}\right\}<+\infty
$$

where $\mathcal{P}$ denotes the set of partitions: $\left\{P_{i}\right\}_{i} \in \mathcal{P}$ if $\sum_{i} P_{i}=\Omega$ i.e., $\cup_{i} P_{i}=\Omega$ and $P_{i} \cap P_{j}=\emptyset$ for $i \neq j$.

We can define a (extended) set function $\bar{w}$ in the following manner,

$$
\forall A \in \mathcal{A}, \bar{w}(A)=\sup \left\{\sum_{i} w\left(P_{i}\right): \sum_{i} P_{i}=A\right\} \in[0, \infty] .
$$

By construction $\bar{w}$ is superadditive and $\bar{w} \geq w$ (see Theorem 3.1 in [8]).

We consider a class of set functions which is wider than the class of subadditive set functions.

A set function is almost subadditive if

$$
\forall A, B \in \mathcal{A}, A B=\emptyset, w(A \cup B) \leq \bar{w}(A)+w(B),
$$

Hence the contribution of $A$ to $B$ can not be greater than the maximal contributions of disjoint subsets of $A$.

Various notions of continuity can be introduced whenever we consider set functions.

$w$ is order-continuous i.e., $w \downarrow \emptyset$, if $w\left(A_{n}\right) \rightarrow 0$ whenever $A_{n} \downarrow \emptyset$.

$w$ is semicontinuous from below, if $\liminf _{n} w\left(A_{n}\right) \geq w(A)$ whenever $A_{n} \uparrow A$.

$w$ is continuous from below, if $\lim _{n} w\left(A_{n}\right)=w(A)$ whenever $A_{n} \uparrow A$.

Their relationships are presented in [9]. For monotone subadditive set functions of bounded sum these three notions are equivalent.

Let $\mu, w$ be set functions. $\mu$ dominates $w$ i.e., $\mu>>w$, if for all $A \in \mathcal{A}$, $\mu(A)=0 \Rightarrow w(A)=0 . \mu, w$ are equivalent, i.e., $\mu \equiv w$, whenever $\mu>>w$ and $\mu<<w$.

The Radon-Nikodym theorem we shall consider for set functions w.r.t. measures introduce a one-sided Radon-Nikodym derivative.

Definition A.1 Let $w$ be a set function and $\mu$ a measure. $w$ admits a superior Radon-Nikodym derivative w.r.t. $\mu$ if there exists an integrable function $f^{s} \geq 0$ 
that majorizes $w$, i.e. $w \leq f^{s} \mu$,

$$
\forall A \in \mathcal{A}, w(A) \leq \int_{A} f^{s} d \mu
$$

which is minimal i.e.

$$
\forall g \text { integrable, } w \leq g \mu \Rightarrow f^{s} \leq g, \mu-\text { a.e. }
$$

We shall say that $\frac{d w}{d \mu}^{s}$ exists and write $\frac{d w}{d \mu}^{s}=f^{s}$ where no confusion is possible.

Hence, whenever $w$ admits a superior Radon-Nikodym derivative, $w$ is necessarily of bounded sum, order-continuous and dominated by $\mu$.

Theorem: (SEe TheORem 1 IN [9])

Let $w$ be an a.-subadditive set function and $\mu$ a measure. Then, $w$ admits a superior Radon-Nikodym derivative w.r.t. $\mu$ if and only if

$$
w \in B S, w \text { is semicontinuous from below, } \mu>>w
$$

Moreover, $f^{s} \mu \equiv w$ if and only if $w$ is $0-H$.

Then, we may restate the theorem for our purpose

\section{Theorem 1 (Subadditive CASE)}

Let $w$ be a monotone subadditive set function and $\mu$ a measure. Then, $w$ admits a superior Radon-Nikodym derivative w.r.t. $\mu$ if and only if

$$
w \in B S, w \text { is order-continuous, } \mu>>w
$$

Moreover, $f^{s} \mu \equiv w$.

Proof. By definition of the superior Radon-Nikodym derivative the necessity part holds. As for sufficiency, a monotone order-continuous subadditive set function is $0-\mathrm{H}$, almost subadditive and semicontinuous from below, so we may apply Theorem 1 in [9].

We now consider the operation of supremum, that allows one to build a.subadditive set functions from existing ones. Then, we explore how RadonNikodym derivatives are obtained through suprema. This makes the structure of a.-subadditive set functions of bounded sum and semicontinuous from below a sup-lattice which is also boundedly complete. The subset of subadditive set functions is moreover a convex cone and boundedly complete too.

Lemma 1 Let $w_{1}, w_{2}$ be set functions, $w_{1}, w_{2} \in B S$ be a.-subadditive. Then $\max \left\{w_{1}, w_{2}\right\}$ is a.-subadditive of bounded sum. Moreover,

$$
\overline{\max \left\{w_{1}, w_{2}\right\}}=\bar{w}_{1} \vee \bar{w}_{2}
$$

where $\vee$ denotes the supremum for charges. 
Proof : At first we check that $w=\max \left\{w_{1}, w_{2}\right\}$ is of bounded sum.

We have $w \leq w_{1}+w_{2}$, thus $\bar{w} \leq \overline{w_{1}+w_{2}} \leq \bar{w}_{1}+\bar{w}_{2}$. As for a.-subadditivity. Let $A, B \in \mathcal{A}$ with $A \cap B=\emptyset$. Assume $w_{1}(A+B) \leq w_{2}(A+B)$. Then,

$w(A+B)-w(A)=w_{2}(A+B)-w(A) \leq w_{2}(A+B)-w_{2}(A) \leq \bar{w}_{2}(B) \leq \bar{w}(B)$.

Let us prove that $\bar{w}=\bar{w}_{1} \vee \bar{w}_{2}$. We have $w=\max \left\{w_{1}, w_{2}\right\} \leq \max \left\{\bar{w}_{1}, \bar{w}_{2}\right\} \leq$ $\bar{w}_{1} \vee \bar{w}_{2}$. Thus, $\bar{w} \leq \bar{w}_{1} \vee \bar{w}_{2}$ holds. For the other inequality, $w \geq w_{i}$, thus $\bar{w} \geq \bar{w}_{i}$ hence $\bar{w} \geq \bar{w}_{1} \vee \bar{w}_{2}$.

Corollary 1 Let $w_{1}, w_{2}$ be a.-subadditive set functions and $\mu$ a measure such that ${\frac{d w_{1}}{d \mu}}^{s},{\frac{d w_{2}}{d \mu}}^{s}$ exist then

$$
\frac{d \max \left\{w_{1}, w_{2}\right\}^{s}}{d \mu}=\max \left\{{\frac{d w_{1}}{d \mu}}^{s},{\frac{d w_{2}}{d \mu}}^{s}\right\}
$$

Proof : Since ${\frac{d w_{1}}{d \mu}}^{s},{\frac{d w_{2}}{d \mu}}^{s}$ exist, $w_{1}, w_{2}$ are semicontinuous from below, of bounded sum and dominated by $\mu$. Hence $\max \left\{w_{1}, w_{2}\right\}$ is also semicontinuous from below, of bounded sum and dominated by $\mu$. Moreover according to Lemma 1 , $\max \left\{w_{1}, w_{2}\right\}$ is a.-subadditive, thus its superior Radon-Nikodym derivative exists.

Let $A \in \mathcal{A}$, with $w_{1}(A) \geq w_{2}(A)$ for instance,

$$
\max \left\{w_{1}(A), w_{2}(A)\right\}=w_{1}(A) \leq{\frac{d w_{1}}{d \mu}}^{s} \mu(A) \leq \max \left\{{\frac{d w_{1}}{d \mu}}^{s},{\frac{d w_{2}}{d \mu}}^{s}\right\} \mu(A)
$$

thus $\mu$ a.e.,

$$
\frac{d \max \left\{w_{1}, w_{2}\right\}^{s}}{d \mu} \leq \max \left\{\frac{d w_{1}^{s}}{d \mu}, \frac{d w_{2}^{s}}{d \mu}\right\}
$$

For the reverse inequality, since $\max \left\{w_{1}, w_{2}\right\} \geq w_{1}$ it comes

$$
\frac{d \max \left\{w_{1}, w_{2}\right\}^{s}}{d \mu} \mu \geq w_{1}
$$

thus $\mu$ a.e.,

$$
\frac{d \max \left\{w_{1}, w_{2}\right\}^{s}}{d \mu} \geq{\frac{d w_{1}}{d \mu}}^{s}
$$

Lemma 1 can be extended to an arbitrary collection of a.-subadditive set functions as soon as it is bounded from above,

Lemma 2 Let $\left\{w_{\alpha}\right\}_{\alpha \in I}$ be a set of a.-subadditive set functions. If there exists a set function $\psi$ of bounded sum such that $w_{\alpha} \leq \psi$ then $\sup _{I} w_{\alpha}$ is a.-subadditive of bounded sum. Moreover,

$$
\overline{\sup _{I} w_{\alpha}}=\vee_{I} \bar{w}_{\alpha}
$$


Proof : Let $\psi$ be of bounded sum. Since $\sup _{I} w_{\alpha} \leq \psi, \sup _{I} w_{\alpha}$ is of bounded sum.

As for a.-subadditivity. Let $A, B \in \mathcal{A}$ with $A \cap B=\emptyset$ and $\epsilon>0$. There is an $\alpha(\epsilon) \in I$ such that,

$\sup _{I}\left[w_{\alpha}(A+B)-w_{\alpha}(B)\right]-\epsilon<w_{\alpha(\epsilon)}(A+B)-w_{\alpha(\epsilon)}(B) \leq \overline{w_{\alpha(\epsilon)}}(A) \leq \overline{\sup _{I} w_{\alpha}}(A)$

thus

$$
\sup _{I} w_{\alpha}(A+B)-\sup _{I} w_{\alpha}(B)-\epsilon<\overline{\sup }_{I} w_{\alpha}(A) .
$$

Since $\overline{\sup _{I} w_{\alpha}}$ is additive and $\overline{\sup _{I} w_{\alpha}} \geq \bar{w}_{\alpha}$ for all $\alpha \in I$, thus $\left\{\bar{w}_{\alpha}: \alpha \in I\right\}$ admits an upper bound. Let $Q$ be an upper bound. For $A \in \mathcal{A}, \epsilon>0$ there is a partition $\left\{A_{i}: i=1, \ldots, n\right\}$ of $A$,

$$
\underset{I}{\sup _{I} w_{\alpha}}(A)-\frac{\epsilon}{2}<\sum_{i=1}^{n} \sup _{I} w_{\alpha}\left(A_{i}\right)
$$

And there are some $\alpha_{i} \in I$ such that

$$
\sup _{I} w_{\alpha}\left(A_{i}\right)-\frac{\epsilon}{2 n}<w_{\alpha_{i}}\left(A_{i}\right)
$$

thus,

$$
\overline{\sup }_{I} w_{\alpha}(A)-\epsilon<\sum_{i=1}^{n} \sup _{I} w_{\alpha}\left(A_{i}\right)-\frac{\epsilon}{2}<\sum_{i=1}^{n} w_{\alpha_{i}}\left(A_{i}\right) \leq \sum_{i=1}^{n} \bar{w}_{\alpha_{i}}\left(A_{i}\right) \leq Q(A)
$$

Since $\epsilon$ is arbitrary chosen, $\overline{\sup _{I} w_{\alpha}} \leq Q$. Hence the least upper bound is precisely given by $\overline{\sup _{I} w_{\alpha}}$.

In particular this shows that $\sup _{I} w_{\alpha}$ is dominated by an additive set function namely $\overline{\sup _{I} w_{\alpha}}$ (which is $\sigma$-additive as soon as the $w_{\alpha}$ 's are lower semicontinuous from below, see Corollary 2). If we consider measures $P_{\alpha}$ 's then $\vee_{I} P_{\alpha}$ exists if and only if the $P_{\alpha}$ 's are bounded from above by a set function of bounded sum, moreover this supremum is equal to $\overline{\sup _{I} P_{\alpha}}$. In particular this shows that the space of bounded charges (countable additive set functions) is boundedly complete (see p. 29, 50 in [2]).

Corollary 2 Let $\left\{w_{\alpha}\right\}_{\alpha \in I}$ be a set of a.-subadditive semicontinuous from below set functions, $\mu$ a measure and $\psi$ a set function of bounded sum such that $w_{\alpha} \leq \psi$. If $w_{\alpha}<<\mu$ for all $\alpha \in I$ then

$$
\frac{d \sup _{I} w_{\alpha}^{s}}{d \mu}=\vee_{I} \frac{d w_{\alpha}^{s}}{d \mu}
$$

Proof : Since $w_{\alpha}<<\mu$ for all $\alpha \in I$ it comes $\sup _{I} w_{\alpha}<<\mu \cdot \sup _{I} w_{\alpha}$ is of bounded sum since $\psi$ is. $\sup _{I} w_{\alpha}$ is a.-subadditive according to Lemma 2 . 
Let us check for semicontinuity from below. Let $A_{n} \uparrow A$ and $\epsilon>0$. There exists $\alpha_{\epsilon} \in I$ s.t.

$$
\sup _{I} w_{\alpha}(A)-\epsilon<w_{\alpha_{\epsilon}}(A) \leq \liminf _{n} w_{\alpha_{\epsilon}}\left(A_{n}\right) \leq \liminf _{n} \sup _{I} w_{\alpha}\left(A_{n}\right)
$$

Applying Theorem 1 in [9] and Lemma 2 gives

$$
\frac{d \sup _{I} w_{\alpha}^{s}}{d \mu}=\frac{d \overline{\sup _{I} w_{\alpha}}}{d \mu}=\frac{d \vee_{I} \overline{w_{\alpha}}}{d \mu}
$$

Let us endow $\mathcal{L}_{+}^{1}(\mu)$ with the $\mu$-a.e. preorder i.e., $f \geq g \mu$-a.e..

The set of superior Radon-Nikodym derivatives $\left\{\frac{d w_{\alpha} s}{d \mu}: \alpha \in I\right\}$ is clearly dominated by $\frac{d \sup _{I} w_{\alpha}}{d \mu} s$.

Let $g \in \mathcal{L}_{+}^{1}(\mu)$, with $g \geq \frac{d w_{\alpha} s}{d \mu} \mu$-a.e. for all $\alpha \in I$. Then $g \mu \geq w_{\alpha}$ for all $\alpha \in I$. Thus, $g \mu \geq \sup _{I} w_{\alpha}$ and it comes by minimality of the superior Radon-Nikodym derivative,

$$
g \geq \frac{d \sup _{I} w_{\alpha}^{s}}{d \mu}
$$

so $\frac{d \sup _{I} w_{\alpha}}{d \mu} s$ is a least upper bound of $\left\{\frac{d w_{\alpha} s}{d \mu}: \alpha \in I\right\}$.

As a consequence of Corollary 2 we have a direct way to guarantee the existence of suprema in $c a^{+}(\mu)$.

Property: Consider $\mathrm{ca}^{+}(\mu)=\left\{\nu \in c a^{+}: \nu<<\mu\right\}$ and let $\left\{P_{\alpha}\right\}_{I} \subset c a^{+}(\mu)$. If $Q$ is a measure such $Q \geq P_{\alpha}$ then there exists a measure $R \geq P_{\alpha}$ with $R<<\mu$. Hence, the supremum of $\left\{P_{\alpha}\right\}_{I} \subset c a^{+}(\mu)$ exists in $c a^{+}(\mu)$ if and only if it exists in $\mathrm{Ca}^{+}$and these suprema are equal.

Proof : Let $Q \geq P_{\alpha}$, according to Lemma $2, \vee_{I} P_{\alpha}=\overline{\sup _{I} P_{\alpha}}$. Now since $\mu>>P_{\alpha}$, for all $\alpha \in I$ it comes $\mu>>\sup _{I} P_{\alpha}$, thus $\mu>>\vee_{I} P_{\alpha}$. Take $R=\vee_{I} P_{\alpha}$. Assume $\left\{P_{\alpha}\right\}_{I} \subset \mathrm{ca}^{+}(\mu)$ admits a supremum in $\mathrm{ca}^{+}$. The supremum it given by $Q=\vee_{I} P_{\alpha}$. Thus there exists $R \geq P_{\alpha}$ with $R<<\mu$. So $R \geq \vee_{I} P_{\alpha}$, and it comes that $\vee_{I} P_{\alpha}<<\mu$ i.e. $\vee_{I} P_{\alpha} \in c a^{+}(\mu)$. And since $\vee_{I} P_{\alpha}$ is a least upper bound in $c a^{+}$it is also a smaller bound than any upper bound in $c a^{+}(\mu)$, so $\vee_{I} P_{\alpha}$ is also the supremum in $\mathrm{ca}^{+}(\mu)$.

As for the converse, assume the supremum of $\left\{P_{\alpha}\right\}_{I} \subset c a^{+}(\mu)$ exists and let us denote it by $P$. Since $P_{\alpha} \leq P$ we have $P_{\alpha} \leq \vee_{I} P_{\alpha} \leq P$ and $\vee_{I} P_{\alpha}<<\mu$ since $P \in c a^{+}(\mu)$, thus $\vee_{I} P_{\alpha}$ is an upper bound in $c a^{+}(\mu)$. By minimality of $P$, it comes $P=\vee_{I} P_{\alpha}$, which is the supremum in $\mathrm{Ca}^{+}$.

\section{B Proofs}




\section{B.1 The Positive result}

We may now give a proof of the existence of an information density function in a decision making problem under uncertainty and the way the information density function can be obtained.

\section{Proof.}

We shall check that the conditions for the existence of a superior Radon-Nikodym derivative are met and then give an explicite formula to compute it.

At first, we check that the set function Cap, where

$$
\forall A \in \mathcal{A}, \operatorname{Cap}(A)=\sup _{S} \int_{A} u(s, \omega) d \mu(\omega)
$$

is well defined, monotone and subadditive.

- Cap is a well defined set function.

Since $0 \leq \operatorname{Cap}(A) \leq M \mu(A) \leq M<\infty$ and $\operatorname{Cap}(\emptyset)=0$.

- Cap is monotone.

Let $A \subset B, A, B \in \mathcal{A}$, then $\operatorname{Cap}(A) \leq \operatorname{Cap}(B)<\infty$ since $u(s,) \geq$.0 .

- Cap is subadditive.

Let $A \subset B, A, B \in \mathcal{A}$ with $A \cap B=\emptyset$. There exists $s_{\epsilon} \in S$ such that,

$$
\begin{gathered}
\operatorname{Cap}(A+B)-\epsilon<\int_{A+B} u\left(s_{\epsilon}, .\right) d \mu=\int_{A} u\left(s_{\epsilon}, .\right) d \mu+\int_{B} u\left(s_{\epsilon}, .\right) d \mu \\
\leq \operatorname{Cap}(A)+\operatorname{Cap}(B)
\end{gathered}
$$

since $\epsilon$ can be arbitrary chosen close to $0, \operatorname{Cap}(A+B) \leq \operatorname{Cap}(A)+\operatorname{Cap}(B)$ holds.

Let us prove that Cap satisfies the sufficient conditions of Theorem 1.

Since Cap $\leq M \mu$ and $\mu$ is $\sigma$-additive, it holds

- Cap is order-continuous.

Let $A_{n} \downarrow \emptyset$. It comes, $0 \leq \lim _{n} \operatorname{Cap}\left(A_{n}\right) \leq M \lim _{n} \mu\left(A_{n}\right)=0$.

- Cap is of bounded sum.

Let $\left\{A_{i}\right\}_{i=1}^{n}$ be a partition. Then, $\sum_{i=1}^{n} \operatorname{Cap}\left(A_{i}\right) \leq M \sum_{i=1}^{n} \mu\left(A_{i}\right)=M \mu(\Omega)<\infty$.

- Cap is dominated by $\mu$.

We have Cap $<<M \mu$ with $M>0$ thus Cap $<<\mu$.

So Cap fulfills the hypothesis of Theorem 1. Thus Cap admits a superior Radon-Nikodym derivative w.r.t. $\mu$. Take $U=\frac{d \mathrm{Cap}^{s}}{d \mu}$. By definition $I=\overline{\mathrm{Cap}}$, so the information set function (measure) can be factorized by $U$ through $\mu$, i.e.,

$$
\frac{d I}{d \mu}=\frac{d \overline{\mathrm{Cap}}}{d \mu}=U
$$

and by minimality of the superior Radon-Nikodym derivative, for any integrable $V: \Omega \longrightarrow \mathbb{R}^{+}$,

$$
\forall A \in \mathcal{A}, \operatorname{Cap}(A) \leq \int_{A} V(\omega) d \mu(\omega) \Rightarrow U \leq V, \mu \text { a.s. }
$$


Moreover since Cap is monotone, thus null hereditary, it holds for all $A \in \mathcal{A}$,

$$
\operatorname{Cap}(A)=0 \Longleftrightarrow I(A)=\int_{A} U d \mu=0 \Longleftrightarrow \mu(A \cap\{U>0\})=0 .
$$

Secondly, to obtain explicitely the Radon-Nikodym derivative formula we apply directely Corollary 2.

Take for index set $I=S, \psi=M \mu$ and $w_{\alpha}=m_{s}$ where $m_{s}()=.\int_{(.)} u(s, \omega) d \mu(\omega)$. So,

$$
\sup _{I} w_{\alpha}=\sup _{S} m_{s}=\text { Cap. }
$$

And it comes

$$
U=\frac{d \mathrm{Cap}^{s}}{d \mu}=\bigvee_{s \in S} \frac{d m_{s}}{d \mu}=\bigvee_{s \in S} u(s, .)
$$

\section{B.2 The countable case}

Let us show that the information density function is precisely $V=\sup _{S} u(s,$. whenever $\Omega=\mathbb{N}, \mathcal{A}=2^{\mathrm{N}}$ with $\mu(\omega)>0$.

Proof.

Since $\mathcal{A}=2^{\mathrm{N}}, V$ is measurable and for all $A \subset \mathbb{N}$,

$$
\operatorname{Cap}(A)=\sup _{S} \int_{A} u(s, .) d \mu \leq \int_{A} \sup _{S} u(s, .) d \mu \leq \int_{A} V d \mu .
$$

So $V$ majorizes Cap.

It remains to check that $V$ is minimal.

Let $f$ be a function such that Cap $\leq f \mu$. In particular, for all $\omega \in \Omega$

$$
\operatorname{Cap}(\{\omega\}) \leq f(\omega) \mu(\omega) .
$$

But,

$$
\operatorname{Cap}(\{\omega\})=_{\operatorname{def}} V(\omega) \mu(\omega) \text { and } \mu(\omega)>0
$$

thus $V(\omega) \leq f(\omega)$. So $V$ is the superior Radon-Nikodym derivative of Cap w.r.t. $\mu$.

\section{B.3 AWTP}

Let us prove that the AWTP, $\Phi_{u}(m, \Pi)=\frac{I_{u_{2}}(\Omega)-\mathrm{Cap}_{u_{2}}(\Pi)}{u_{1}^{\prime}(m)}$, is an upper bound to the DM's willingness to pay for any $\Pi^{\prime}$.

\section{Proof.}

An additional information at cost $p$ is worthwhile whenever

$$
u_{1}(m-p)+\operatorname{Cap}_{u_{2}}\left(\Pi \vee \Pi^{\prime}\right) \geq u_{1}(m)+\operatorname{Cap}_{u_{2}}(\Pi)
$$


thus,

$$
u_{1}(m)-u_{1}(m-p) \leq \operatorname{Cap}_{u_{2}}\left(\Pi \vee \Pi^{\prime}\right)-\operatorname{Cap}_{u_{2}}(\Pi) \leq I_{u_{2}}(\Omega)-\operatorname{Cap}_{u_{2}}(\Pi)
$$

Moreover $u_{1}$ is concave and differentiable at $m$, thus

$$
u_{1}(m)-u_{1}(m-p) \geq-u_{1}^{\prime}(m)((m-p)-m)=u_{1}^{\prime}(m) p .
$$

Since $u_{1}^{\prime}(m)>0$ it comes,

$$
p \leq \frac{I_{u_{2}}(\Omega)-\operatorname{Cap}_{u_{2}}(\Pi)}{u_{1}^{\prime}(m)}=\Phi_{u}(m, \Pi) .
$$

Hence the maximal price the DM is ready to pay for any $\Pi^{\prime}$ is bounded from above by $\Phi_{u}(m, \Pi)$.

Let us check that $\Phi_{u}(m, A)$ is the maximal price the DM could pay for being more informed whenever A occurs.

\section{Proof.}

Let $A \in \mathcal{A}$. Consider the partitions $\Pi=\left\{A_{1}, \ldots, A_{K}\right\}$ with $A=A_{k}$ for some $k$ and $\Pi^{\prime}=\left\{B_{1}, \ldots, B_{J}\right\}$.

Let us consider the following information structure

$$
\Pi \triangleright_{A} \Pi^{\prime}=\left\{A_{l}: l \neq k\right\} \cup\left\{A \cap B_{j}: j=1, \ldots, J\right\} .
$$

In otherwords the set $A$ is "decomposed" over $\Pi^{\prime}$, whereas the other sets $A_{l}$ remain inchanged, hence the DM has a finer information structure than $\Pi$ and this additional information is gained whenever $A$ occurs by means of $\Pi^{\prime}$, so the DM is offered $\Pi^{\prime}$ contingently.

This contingent information at $\operatorname{cost} p_{A}$ is worthwhile whenever,

$$
u_{1}\left(m-p_{A}\right)+\operatorname{Cap}_{u_{2}}\left(\Pi \triangleright_{A} \Pi^{\prime}\right) \geq u_{1}(m)+\operatorname{Cap}_{u_{2}}(\Pi)
$$

thus,

$$
\begin{aligned}
& u_{1}(m)-u_{1}\left(m-p_{A}\right) \leq \operatorname{Cap}_{u_{2}}\left(\Pi \triangleright_{A} \Pi^{\prime}\right)-\operatorname{Cap}_{u_{2}}(\Pi) \\
= & \sum_{j=1}^{J} \operatorname{Cap}_{u_{2}}\left(A \cap B_{j}\right)-\operatorname{Cap}_{u_{2}}(A) \leq I_{u_{2}}(A)-\operatorname{Cap}_{u_{2}}(\Pi)
\end{aligned}
$$

Following the same lines than the previous proof, it comes

$$
p_{A} \leq \frac{I_{u_{2}}(A)-\operatorname{Cap}_{u_{2}}(\Pi)}{u_{1}^{\prime}(m)}=\Phi_{u}(m, A) .
$$

Hence the maximal price the DM is ready to pay for $\Pi^{\prime}$ whenever $A$ occurs is bounded from above by $\Phi_{u}(m, A)$.

Let us prove that $\Phi_{u}(m, \Pi)$ can decomposed setwise with respect to $\Pi$. 


\section{Proof.}

Let $\Pi=\left\{A_{1}, \ldots, A_{K}\right\}$. Since $I_{u_{2}}$ is additive, $I_{u_{2}}(\Omega)=\sum_{k=1}^{K} I_{u_{2}}\left(A_{k}\right)$ and by definition $\operatorname{Cap}_{u_{2}}(\Pi)=\sum_{k=1}^{K} \operatorname{Cap}_{u_{2}}\left(A_{k}\right)$, thus

$$
\Phi_{u}(m, \Pi)=\frac{I_{u_{2}}(\Omega)-\operatorname{Cap}_{u_{2}}(\Pi)}{u_{1}^{\prime}(m)}=\sum_{k=1}^{K} \frac{I_{u_{2}}\left(A_{k}\right)-\operatorname{Cap}_{u_{2}}\left(A_{k}\right)}{u_{1}^{\prime}(m)}=\sum_{k=1}^{K} \Phi_{u}\left(m, A_{k}\right) .
$$

Let us prove that the quantities $\Phi_{u}(m, \Pi), \Phi_{u}(m, A)$ are cardinal.

\section{Proof.}

The utility function $(m,(s, \omega)) \mapsto u(m,(s, \omega))$ is defined up to a positive linear transformation. Let $a>0, b \in \mathbb{R}$ and consider

$$
v=a u+b=a\left(u_{1}+u_{2}\right)+b=\left(a u_{1}+b\right)+a u_{2}=v_{1}+v_{2} .
$$

We have, $\left(v_{1}\right)^{\prime}=\left(a u_{1}+b\right)^{\prime}=a u_{1}^{\prime}$ and $I_{v_{2}}(\Omega)-\operatorname{Cap}_{v_{2}}(\Pi)=a\left(I_{u_{1}}(\Omega)-\operatorname{Cap}_{u_{1}}(\Pi)\right)$, thus

$$
\Phi_{v}(m, \Pi)=\frac{I_{v_{2}}(\Omega)-\operatorname{Cap}_{v_{2}}(\Pi)}{v_{1}^{\prime}(m)}=\frac{a}{a} \frac{I_{u_{2}}(\Omega)-\operatorname{Cap}_{u_{2}}(\Pi)}{u_{1}^{\prime}(m)}=\Phi_{u}(m, \Pi) .
$$

The proof is similar for $\Phi_{u}(m, A)$.

Let us prove that for all $\left(m, \Pi, \Pi^{\prime}\right)$, it holds

$$
W T A \geq \Phi_{u}(m, \Pi)-\Phi_{u}\left(m, \Pi \vee \Pi^{\prime}\right) \geq W T P .
$$

\section{Proof.}

Indeed, a DM, with initial wealth and information $\left(m, \Pi \vee \Pi^{\prime}\right)$, is willing to abandon some information $\Pi^{\prime}$ at some price $p$ if,

$$
u_{1}(m)+\operatorname{Cap}_{u_{2}}\left(\Pi \vee \Pi^{\prime}\right) \leq u_{1}(m+p)+\operatorname{Cap}_{u_{2}}(\Pi)
$$

or

$$
\begin{aligned}
u_{1}(m+p)-u_{1}(m) & \geq \operatorname{Cap}_{u_{2}}\left(\Pi \vee \Pi^{\prime}\right)-\operatorname{Cap}_{u_{2}}(\Pi) \\
& =u^{\prime}(m)\left[\Phi_{u}(m, \Pi)-\Phi_{u}\left(m, \Pi \vee \Pi^{\prime}\right)\right]
\end{aligned}
$$

and since $u_{1}$ is concave it comes

$$
p \geq \Phi_{u}(m, \Pi)-\Phi_{u}\left(m, \Pi \vee \Pi^{\prime}\right)
$$

thus the willingness to accept is greater than $\Phi_{u}(m, \Pi)-\Phi_{u}\left(m, \Pi \vee \Pi^{\prime}\right)$.

Similarely, a DM with initial wealth and information $(m, \Pi)$ is willing to pay some additional information $\Pi^{\prime}$ at some price $p$ if,

$$
u_{1}(m-p)+\operatorname{Cap}_{u_{2}}\left(\Pi \vee \Pi^{\prime}\right) \geq u_{1}(m)+\operatorname{Cap}_{u_{2}}(\Pi)
$$

or

$$
\begin{aligned}
u_{1}(m-p)-u_{1}(m) & \geq \operatorname{Cap}_{u_{2}}(\Pi)-\operatorname{Cap}_{u_{2}}\left(\Pi \vee \Pi^{\prime}\right) \\
& =u^{\prime}(m)\left[\Phi_{u}\left(m, \Pi \vee \Pi^{\prime}\right)-\Phi_{u}(m, \Pi)\right]
\end{aligned}
$$


and since $u_{1}$ is concave it comes

$$
p \leq \Phi_{u}(m, \Pi)-\Phi_{u}\left(m, \Pi \vee \Pi^{\prime}\right)
$$

thus the willingness to pay is smaller than $\Phi_{u}(m, \Pi)-\Phi_{u}\left(m, \Pi \vee \Pi^{\prime}\right)$.

\section{References}

[1] R.J. Aumann, Subjectivity and Correlation in Randomized Strategies, Journal of Mathematical Economics 1 (1974) 67-96.

[2] K.P.S. Bhaskara Rao and M. Bhaskara Rao. Theory of charges. Academic Press, New York, 1983.

[3] P.C. Fishburn. Utility Theory for Decision Making. Wiley, New York, 1970.

[4] I. Gilboa, E. Lehrer, The value of information - An axiomatic approach, Journal of Mathematical Economics 20 (1991) 443-459.

[5] J. Kennan, The existence of expected utility maximizing decisions when utility is unbounded, Econometrica 49 (1981) 215-18.

[6] I. La Valle, On Cash Equivalence and Information Evaluation in Decision under and Uncertainty, part I, II and III. Journal of The American Statistical Association, 63 (1968) 252-290.

[7] E. Lehrer and D. Rosenberg, What restrictions do Bayesian games impose on the value of information?, Journal of Mathematical Economics 42 (2006) 343-357.

[8] E. Pap. Null-additive set functions. Kluwer Academic Publishers, Dordrecht, 1995.

[9] Y. Rébillé, A Radon-Nikodym theroem for almost subadditive set functions, working paper LEMNA (2009).

[10] L. J. Savage. The foundation of statistics. New York, Wiley, 1954. 